\title{
CONSERVATION LAWS IN THE HIERARCHICAL MODEL
}

\author{
H. VAN BEYEREN, G. GALLAVOTTI and H. KNOPS
}

Instituut voor Theoretische Fysika, Universiteit Nijmegen, Nijmegen, The Netherlands

Received 3 July 1974

\section{Synopsis}

An exposition of the renormalization-group equations for the hierarchical model is given. Attention is drawn to some properties of the spin distribution functions which are conserved under the action of the renormalization group.

1. Introduction. In the framework of the recent theories of the critical point a universality class is associated with a fixed point of a renormalization transformation (the correspondence being such that many fixed points and many renormalization transformations may give rise to the same universality class). The problem is to find, given a model and a renormalization transformation, which is the fixed point associated with its critical behaviour.

In this note we show with some examples how one might be helped in this search by some "conservation laws". We shall study only the hierarchical models where a simple renormalization group can be considered. We shall show that the fixed points associated with certain models will have to obey restrictions corresponding to the three "conservation laws" that we shall find.

Most of this paper, in order to provide a motivation to the remarks of section 5, deals with an exposition of known results and ideas about recursion formulas and the $\varepsilon$-expansion. The reader is supposed to have some familiarity with the basic ideas and assumptions of Wilson's theory of the critical point (which is not described here).

2. The hierarchical model. Consider a 1-dimensional lattice $Z^{+}=(1,2, \ldots)$ and think of it as built up with adjacent blocks of $2^{p}$ sites $(p=0,1, \ldots)$. The $r$ th block of $2^{p}$ sites will be denoted by $(r, p)$. So

$$
(r, p)=\left\{(r-1) 2^{p}+1,(r-1) 2^{p}+2, \ldots, r 2^{p}\right\} .
$$


On each site $i$ of the lattice sits a spin $\sigma_{i} \in(-\infty, \infty)$. We define

$$
\begin{aligned}
& M_{r, p}=\sum_{i \in(r, p)} \sigma_{i}, \\
& S_{r, p}=2^{-p / 2}\left(M_{2 r-1, p-1}-M_{2 r, p-1}\right)
\end{aligned}
$$

and then define the hamiltonian for a system of $2^{L}$ spins as ${ }^{1-3}$ )

$$
H_{L}\left(\sigma_{1}, \ldots, \sigma_{2^{L}}\right)=\sum_{p=1}^{L} B_{p} \sum_{m=1}^{2 L-p} S_{m, p}^{2}
$$

where the couplings $B_{p}$ are given by

$$
B_{p}=\frac{1}{2} B\left(2^{1-\alpha}\right)^{p-1} \text {, }
$$

with $B>0$ and $1<\alpha<2$. This interaction is a nontranslationally invariant version of the long-range one-dimensional Ising model with potential $\varphi(R)=J R^{-{ }^{1}}$ ).

A "model" is defined by the sequence $\left\{B_{p}\right\}_{1}^{\infty}$ and by the "free spin distribution" $\Pi^{(0)}(\sigma)$ which describes the isolated spins when $B=0$. So the partition function of the model $\left(\left\{B_{p}\right\}_{1}^{\infty}, \Pi^{(0)}\right)$ is

$$
Z_{L}\left(\left\{B_{p}\right\}_{1}^{\infty}, \Pi^{(0)}\right)=\int \mathrm{e}^{-\beta H_{L}\left(\sigma_{1}, \ldots, \sigma_{2 L}\right)} \prod_{i=1}^{2^{L}} \Pi^{(0)}\left(\sigma_{i}\right) \mathrm{d} \sigma_{i} .
$$

The model $\left(\left\{B_{p}\right\}_{1}^{\infty}, \Pi^{(0)}\right)$ with $\Pi^{(0)}(\sigma)=\frac{1}{2}[\delta(\sigma+1)+\delta(\sigma-1)]$ will be called the Ising hierarchical model.

We shall always assume that, at least in the interesting region of $\beta$ the above integral is finite and $\ln Z$ behaves extensively when $L \rightarrow \infty$ [this means that $\Pi^{(0)}(\sigma)$ is not slowly decreasing at infinity].

We define the block spin variable

$$
\nu=M_{1, L^{-\frac{1}{2} \alpha L},}
$$

and call $\Pi^{(L)}$ its probability distribution with respect to the Boltzmann-Gibbs distribution associated with $H_{L}$. In other words

$$
\Pi^{(L)}(v)=\frac{\int \mathrm{e}^{-\beta H_{L}\left(\sigma_{1}, \ldots, \sigma_{2 L}\right)} \delta\left(2^{-\frac{1}{2} \alpha L} M_{1, L}-v\right) \prod_{i=1}^{2^{L}} \Pi^{(0)}\left(\sigma_{i}\right) \mathrm{d} \sigma_{i}}{Z_{L}\left(\left\{B_{p}\right\}_{1}^{\infty}, \Pi^{(0)}\right)}
$$

Then the following renormalization transformation holds:

$$
Z_{L}\left(\left\{B_{p}\right\}_{1}^{\infty}, \Pi^{(0)}\right)=A_{R}^{2^{L-R}} Z_{L-R}\left(\left\{B_{p}\right\}_{1}^{\infty}, \Pi^{(R)}\right),
$$

where

$$
A_{R}=\left(2^{\alpha / 2}\right)^{2^{R}-1} \int K_{\beta}^{R} \cdot \Pi^{(0)}(v) \mathrm{d} v
$$


and, if $\zeta=2^{\frac{1}{2} x-1}$, the operator $K_{\beta}$ is given by

$$
K_{\beta} \cdot \Pi(v)=\int \Pi(\zeta v+y) \Pi(\zeta v-y) \mathrm{e}^{-\beta B y^{2}} \mathrm{~d} y .
$$

The reader can check the above formulae by a direct calculation or by extracting them out of refs. 1-3 where they are proven in detail (with a different notation).

Furthermore it is also true that

$$
\Pi^{(R)}(v)=K_{\beta}^{(R)} \cdot \Pi^{(0)}(v) / \text { normalisation, }
$$

which is the Baker recursion formula (see ref. 2).

3. Fixed point and tangent space. We now assume that for $\beta=\beta_{\mathfrak{c}}$, a model $\left(\left\{B_{p}\right\}_{1}^{\infty}, \Pi^{(0)}\right)$ has a phase transition and that furthermore the limit

$$
\lim _{L \rightarrow \infty} \frac{K_{\beta_{c}}^{L} \cdot \Pi^{(0)}(v)}{K_{\beta_{c}}^{L} \cdot \Pi^{0}(0)}=\Pi^{(\infty)}(v)
$$

exists in some sense. Then $\Pi^{\infty}$ should be a "fixed point" for $K_{\beta_{\mathrm{c}}}$, i.e., $K_{\beta_{\mathrm{c}}} \cdot \Pi^{(\infty)}$ $=\lambda \Pi^{(\infty)}$ for some $\lambda$. We, furthermore, assume that, if $\beta$ close to $\beta_{\mathrm{c}}$, the ratio

$$
r(v)=\frac{K_{\beta}^{L} \cdot \Pi^{(0)}(v)}{K_{\beta}^{L} \cdot \Pi^{(0)}(0)} \mid \frac{\Pi^{(\infty)}(v)}{\Pi^{(\infty)}(0)}=\exp [\delta(v)],
$$

is for some value $L=L_{\beta-\beta_{c}}$ very close to one in the sense that the function $\delta$ belongs to a neighbourhood of the origin of some linear space of functions which we denote by $\partial K^{\ddagger}$.

If the above assumptions are indeed realized (which may be false or difficult to verify for a given model) Wilson's theory provides a description of the neighbourhood of the critical point in terms of $\Pi^{(\infty)}$ and of the linearization $T_{\beta}$ of the operator $K_{\beta}$ around $\Pi^{(\infty)}$ on the space $\partial K$ :

$$
K_{\beta} \cdot \Pi^{(\infty)} \exp (\delta)=\lambda \Pi^{(\infty)} \exp \left[T_{\beta} \delta+\mathcal{O}\left(\delta^{2}\right)\right] .
$$

In the case of an ordinary critical point the operator $T_{\beta}$ should have, on $\partial K$, a (discrete) spectrum with all the nonconstant eigenfunctions but one having eigenvalues less than $1^{4}$ ).

₹ The reader will notice that the assumptions are not formulated in a way which would satisfy a mathematician. Actually the exact meaning to be given to them is not quite understood in general. Essentially one means that the assumptions should be made precise to allow the calculations below. An example of a set of sufficient assumptions is in the paper by Bleker and Sinai ${ }^{1}$ ). 
A universality class in the frame of the hierarchical models with a given value of the parameter $\alpha$, is associated with the largest eigenvalue $\lambda_{1}$ of $T_{\beta_{\mathrm{c}}}$ (with nonconstant eigenfunction) i.e. all the above models which at $\beta_{c}$ give rise to a couple $\left(\Pi^{(\infty)}, \partial K\right)$ such that the operator $K_{\beta_{\mathrm{c}}}$ linearized around $\Pi^{(\infty)}$ on $\partial K$ has the same value of $\lambda_{1}$, have the same critical exponents.

For instance there is a trivial fixed point $\Pi^{(\infty)}(v) \equiv 1$. If $\partial K$ is taken to be the space of the square-integrable functions (with respect to a suitable weight) the spectrum of $T_{\beta}$ can be easily studied; it is discrete and the eigenvalues relative to nonconstant eigenfunctions are (Bleker and Sinai $\left.\left.{ }^{3}\right)\right)$ :

$$
\lambda_{1}=2 \zeta^{2}, \quad \lambda_{2}=2 \zeta^{4}, \quad \lambda_{3}=2 \zeta^{6}, \ldots
$$

In particular we see that $\lambda_{1}>\lambda_{2}>\lambda_{3}$ and $\lambda_{2}<1$ if $1<\alpha<\frac{3}{2}$.

4. The e-expansion around $\alpha=\frac{3}{2}$. The above discussion makes clear the importance of the correct determination of $\Pi^{(\infty)}$ and $\partial K$ : in fact the spectrum may dramatically depend on their choice. Of great importance is the fact that it has been possible to find some models for which the above assumptions and Wilson's theory could be rigorously verified; (these examples have a $\Pi^{(0)}$ which is rather complicated and $1<\alpha<\frac{3}{2}$; see ref. 3 ).

So far this check of the theory has been possible only for a class of models whose critical point is described by the couple $\left(\Pi^{(\infty)}, \partial K\right)$ introduced as an example at the end of the last section ("gaussian" fixed point).

In the general case, however, little can be said; even if the above assumptions about the existence of $\Pi^{(\infty)}$ and $\partial K$ are accepted for a certain model, it is very hard to judge which is the appropriate couple $\left(\Pi^{(\infty)}, \partial K\right)$. The trouble is that there are, a priori, many fixed points and many tangent spaces that can be associated with the same renormalization transformation $K_{\beta}$.

For instance there is evidence for a fixed point of $K_{\beta}$ of the form

$$
\Pi^{(\infty)}(v)=\exp \left[a v^{2}-b v^{4}+\mathcal{O}\left(\varepsilon^{3} v^{6}\right)\right]
$$

where $\varepsilon=2 \zeta^{4}-1$ and $a, b$ are determined by $\varepsilon$. The evidence is the following: use the r.h.s. of (4.1) as an "ansatz" and apply $K_{\beta}$ to it assuming $a, b$ of order $\varepsilon$. A simple calculation leads to a $\Pi^{\prime}=K_{\beta} \Pi^{(\infty)}$ of the same form but with constants $a^{\prime}$ and $b^{\prime}$ given by

$$
\begin{aligned}
& a^{\prime}=2 \zeta^{2} a-\frac{6 \zeta^{2} b}{\beta B}-\frac{12 \zeta^{2} a b}{(\beta B)^{2}}+\frac{36 \zeta^{2} b^{2}}{(\beta B)^{2}}+\mathcal{O}\left(\varepsilon^{3}\right), \\
& b^{\prime}=2 \zeta^{4} b-\frac{36 \zeta^{4} b^{2}}{(\beta B)^{2}}+\mathcal{O}\left(\varepsilon^{3}\right) .
\end{aligned}
$$


Setting $a^{\prime}=a, b^{\prime}=b$ and observing that for $\varepsilon$ small, $2 \zeta^{4}-1$ is proportional to $\varepsilon$, one finds the fixed-point values

$$
\begin{aligned}
& a(\varepsilon)=\frac{6 \zeta^{2}}{\left(2 \zeta^{2}-1\right) \beta} b(\varepsilon)+\mathcal{O}\left(\varepsilon^{2}\right), \\
& b(\varepsilon)=\frac{\beta^{2}}{36 \zeta^{4}}\left(2 J^{4}-1\right)+\mathcal{O}\left(\varepsilon^{2}\right) .
\end{aligned}
$$

(Another solution is, of course, $a=b=0$.) So the calculation suggests that near $\varepsilon=0$ there is another fixed point which is asymptotically, as $\varepsilon \rightarrow 0$, given by the above formula.

If the space $\partial K$ is taken to be a space which contains $\nu^{2}, \nu^{4}, \ldots$ the above calculations provide evidence that the eigenvalues of the linearized operator $T_{\beta}$ are given by:

$$
2 \zeta^{2}=2^{\frac{1}{2}}\left(1+\frac{1}{2} \varepsilon\right)+\mathcal{O}\left(\varepsilon^{2}\right), \quad 2 \zeta^{4}=1+\varepsilon, \ldots
$$

if one linearizes around $\Pi^{(0)}(v) \equiv 1$ (i.e., $a=b=0$ ), or

$$
2^{\frac{1}{2}}(1+\varepsilon / 6)+\mathcal{O}\left(\varepsilon^{2}\right), \quad 1-\varepsilon+\mathcal{O}\left(\varepsilon^{2}\right), \ldots
$$

if one linearizes around $\Pi^{(\infty)}(\nu)=\exp \left[a(\varepsilon) v^{2}-b(\varepsilon) v^{4} \cdots\right]$. If one assumes that $\partial K$ does not contain $v^{4}$ then the second eigenvalue is missing in both cases.

The reader will no doubt notice that we are treating the spectrum of $T_{\beta}$ and the linear space $\partial K$ in a rather naive way (for instance the statement that $\nu^{4}$ does not belong to $\partial K$ is not very clear since $\partial K$ has not a well-defined topology). The above arguments have, so far, never been made rigorous and the reader should consider them as heuristic considerations.

The above examples explicitly suggest the dependence of the spectrum, and hence of the theory of the critical point of a model, on the couple $\left(\Pi^{(\infty)}, \partial K\right)$. So far the only suggestions about the choice of $\left(I^{(\infty)}, \partial K\right)$ for a given model have come out of perturbation theory. For instance in the Ising hierarchical model the perturbation-theory suggestion is to use $\Pi^{(\infty)}$ of the form $\exp \left(a v^{2}-b v^{4}\right)$ (for $v$ small) and then to linearize for $\delta(v)=a^{\prime} v^{2}-b^{\prime} \nu^{4}$ (for $v$ small). This immediately leads to the result that the critical-point behaviour must be described by the trivial fixed point if $\alpha<\frac{3}{2}$ and by the fixed point discussed above for $\alpha>\frac{3}{2}$. This follows from the fact that the spectral properties required by Wilson's theory are not verified for $\alpha>\frac{3}{2}$ if $K_{\beta}$ is linearized around the trivial fixed point, whereas these properties may be satisfied if $K_{\beta}$ is linearized around the other fixed point (and vice versa for $\alpha<\frac{3}{2}$ ). 
However from a mathematical point of view the problem seems to be rather difficult and it is not even known whether "nontrivial" fixed points really exist nor is it understood any better why the "ansatz" $\Pi^{(\infty)}=\exp \left(a v^{2}-b v^{4}\right)$ should be the right choice for the case of the Ising model.

5. The conservation laws. The above qualitative considerations are meant as a justification of the interest that should be attached to information which could $a$ priori exclude certain possible ansatzes for $\Pi^{(\infty)}$ and $\partial K$ in the investigation of the critical point of a given model. It is in this context that we point out the following "conservation laws".

We assume that the limit which defines $I^{(\infty)}$ in section 3 exists weakly (i.e. $\int_{v_{1}}^{v_{2}} I \Pi^{(\infty)}(v) \mathrm{d} v=\lim _{L \rightarrow \infty} \int_{v_{1}}^{v_{2}} K_{\beta_{\mathrm{c}}}^{L} \cdot \Pi^{(0)}(v) / K_{\beta_{\mathrm{c}}}^{L} \cdot \Pi^{(0)}(0) \mathrm{d} v$ for all $\left.v_{1}, v_{2}\right)$.

I.) Conservation of the bells: if $\Pi^{(0)}$ is a piecewise smooth even function which is monotonic for $v \geqslant 0$ (i.e. if $\Pi^{(0)}$ is "bell shaped") then this property is shared by $K_{\beta}^{L} \cdot \Pi^{(0)}$. The limit $\Pi^{(\infty)}$ is then also bell shaped (but no longer necessarily piecewise smoooth).

II) Conservation of the Fourier type: if $\Pi^{(0)}$ is a positive normalized measure with positive Fourier transform, then $K_{\beta}^{L} \cdot \Pi^{(0)}$ is of the same type. If $\Pi^{(\infty)}$ is also normalizable then $\Pi^{(\infty)}$ is also of positive Fourier type.

III) Conservation of the "Griffiths type": we say that $\Pi^{(0)}(\nu)$ is of "Griffiths type" if

$$
\Pi^{(0)}(v) \simeq \sum_{t_{i} \neq \pm 1} \exp \left(\sum_{i, j} J_{i, j} t_{i} t_{j}\right) \delta\left(\sum_{i=1}^{M} t_{i}-\nu \lambda\right),
$$

for $J_{i, j} \geqslant 0, \lambda>0$, or also if $\Pi^{(0)}$ is a weak limit of a sequence of such functions ${ }^{5}$ ). The property of being of Griffiths type is then conserved under the action of $K_{\beta}$ for $\beta$ such that

$$
0<\beta B / \lambda^{2} \leq 4 J_{i, j}\left(1-1 / 4 \zeta^{2}\right) .
$$

[In case $\Pi^{(0)}(v)$ is obtained from a sequence this relation should hold term by term.] Also $\Pi^{(\infty)}$ is then of Griffiths type.

In the case of the Ising model there is no condition for this last conservation law since $\Pi^{(0)}$ is obtained from only one spin $t$.

There is another way of choosing the initial interactions for a hierarchical model (that of Dyson and Bleker-Sinai ${ }^{1,3}$ )) which leads to a different renormalization-group equation, namely:

$$
\widetilde{K}_{\beta} \cdot \Pi(v)=\mathrm{e}^{\beta D v^{2}} \int \Pi(\zeta v+y) \Pi(\zeta v-y) \mathrm{d} y .
$$

The operator $\tilde{K}_{\beta}$ conserves the Griffiths type for all temperatures; however, the conservation laws I and II are no longer true (but they hold modulo, a gaussian factor, as III does in the case we have considered in this paper).

A proof of the above "laws" is sketched in the appendix. 
6. Concluding remarks. The fixed point which arises in the $\varepsilon$-expansion is neither bell shaped nor of positive Fourier type. We can then use the conservation laws I and II to conclude that the nontrivial fixed point discussed above cannot be relevant for the discussion of the critical point of a model with $a \Pi^{(0)}$ which is either bell shaped or of positive Fourier type. This leads to the conjecture that such models, if describable in terms of Wilson's theory, will be associated with the trivial fixed point $\Pi^{(\infty)}(\nu) \equiv 1$ and described by the eigenvalue $\lambda=2 \zeta^{2}$ even for $\alpha>\frac{3}{2}$ (at least as long as $\alpha-\frac{3}{2}$ is small).

Of course there might be other fixed points compatible with bell-shape or positive Fourier type which are "nontrivial". They may be relevant to the critical point of some model or may become relevant for $\alpha$ large enough. The nontrivial fixed point found with the $\varepsilon$-expansion is compatible with a $\Pi^{(0)}$ of Griffiths type. Hence, as perturbation theory suggests, it is a priori possible to encounter it in the theory of the Ising hierarchical model.

\section{APPENDIX}

In this appendix we sketch a proof of the conservation laws. Consider first the conservation of the bell shape. We recall the definition:

$$
K_{\beta} \cdot \Pi(v)=\int \Pi(\zeta v+y) \Pi(\zeta \nu-y) \mathrm{e}^{-\beta B y^{2}} \mathrm{~d} y .
$$

Suppose now $\Pi(v)$ is even, and monotonic for $v>0$ (i.e. "bell shaped"). The definition of $K_{\beta}$ implies that $K_{\beta} \cdot \Pi(v)$ is even. A few simple substitutions lead to the formula [supposing $\Pi(v)$ is smooth]

$$
\begin{aligned}
& \frac{\mathrm{d}}{\mathrm{d} v} K_{\beta} \cdot \Pi(v) \\
& \quad=\int_{0}^{\infty} \mathrm{e}^{-\beta B y^{2}-\beta B J^{2} v^{2}}\left[\mathrm{e}^{2 \beta B \zeta v y} \Pi(2 \zeta v-y)-\mathrm{e}^{-2 \beta B J v y} \Pi(2 \zeta v+y)\right] \frac{\mathrm{d}}{\mathrm{d} y} \Pi(y) \mathrm{d} y .
\end{aligned}
$$

The fact that $\Pi$ is bell shaped implies that the factor inside the braces is positive (if $v>0$ ) and $\mathrm{d} / \mathrm{d} y \Pi(y) \leq 0$. Hence $\mathrm{d} / \mathrm{d} v\left[K_{\beta} \cdot \Pi(v)\right]$ is negative for $v>0$ and $K_{\beta} \Pi$ is bell shaped. When $\Pi$ is only piecewise smooth and monotonic for $v>0$, one uses the fact that $I I$ can then be weakly approximated by smooth bell-shaped functions. The fact that also the limiting function $\Pi^{(\infty)}$ has to be symmetric and monotonic for $\boldsymbol{\nu}>0$ is evident.

Secondly, the Fourier type: let $\hat{\Pi}(K)$ be the Fourier transform of $\Pi(v)$; it is then easy to check that

$$
\widehat{K_{\beta} \cdot \Pi}(K)=\left(\frac{\pi}{\beta B \zeta}\right)^{\frac{1}{2}} Z^{-1} \int \mathrm{e}^{-y^{2} / \beta B} \hat{\Pi}\left(\frac{K}{2 \zeta}+y\right) \hat{\Pi}\left(\frac{K}{2 \zeta}-y\right) \mathrm{d} y,
$$


where $Z$ is a normalization factor. Consequently $\widehat{K_{\beta} \Pi}$ is positive when $\hat{\Pi}$ is positive. Assuming that $\Pi^{(\infty)}(v)$ is normalized one can prove that $\widehat{K_{\beta}^{M} \cdot I}(K)$ converges to $\hat{\Pi}^{\infty}(K)$, pointwise, hence $I^{(\infty)}$ is also of positive Fourier type.

There is another interesting feature to be learned from this equation. Suppose that there exists for some $\zeta \in\left[2^{-\frac{1}{2}}, 1\right]$ a nontrivial fixed point $\Pi^{(0)}$, which is of positive Fourier type. The fixed-point equation for $\hat{I}^{(0)}$ is the same as the original one, provided one replaces $\beta$ by $\beta^{\prime}=1 / B^{2} \beta$ and $J$ by $J^{\prime}=1 / 2 J$. Hence $\hat{\Pi}^{(0)}$, if positive, is the fixed point of a physical system with $\zeta \in\left[\frac{1}{2}, 2^{-\frac{1}{2}}\right]$, i.e., $\alpha \in[0,1]$. The existence of $\Pi^{(0)}$ of positive Fourier type implies therefore the existence of the free energy of a system with unstable potential $(0<\alpha<1)$ and free spin distribution $\hat{\Pi}^{(0)}$. It is not known whether this possibility can be ruled out for the hierarchical model.

Thirdly the Griffith type: suppose that

$$
\Pi(v) \simeq \sum_{t_{i}= \pm 1} \exp \left(\sum_{i \neq j} J_{i, j} t_{i} t_{j}\right) \delta\left(\sum_{j} t_{j}-\lambda v\right) .
$$

A direct calculation then yields:

$$
\begin{aligned}
K_{\beta} \cdot \Pi(v) \simeq & \sum_{t_{i}, t_{i}^{\prime}= \pm 1} \exp \left(\sum_{i \neq j}\left(J_{i, j}-\beta B / 4 \lambda^{2}\right)\left(t_{i} t_{j}+t_{i}^{\prime} t_{j}^{\prime}\right)\right. \\
& \left.+\sum_{i, j} \beta B / 4 \lambda^{2} t_{i} t_{j}^{\prime}-N \beta B / 2 \lambda^{2}\right) \delta\left(\sum_{j} t_{j}+t_{j}^{\prime}-2 \lambda \zeta v\right) .
\end{aligned}
$$

Repeated application of this formula shows that the coupling $J_{i, J}$ will be diminished by a constant not exceeding the value $\beta B\left(4 \lambda^{2}-\lambda^{2} / \zeta^{2}\right)^{-1}$. The distribution remains therefore of the Griffiths type when $\beta$ fulfils:

$$
\beta B / \lambda^{2} \leq 4 J_{i, j}\left(1-1 / 4 \zeta^{2}\right), \quad i \neq j .
$$

The newly generated couplings between $t_{j}$ and $t_{i}^{\prime}$ remain positive when $\zeta>2^{-\frac{1}{2}}$, which is also the condition required for stability.

\section{REFERENCES}

1) Dyson, F., Comm. math. Phys. 12 (1969) 91.

2) Baker, G., Phys. Rev. B5 (1972) 2622.

3) Bleher, P. and Sinai, J., Comm. math. Phys. 33 (1973) 23.

4) Wilson, K. and Kogut, J., Physics Reports 12 (1974) 75.

5) Simon, B. and Griffiths, R., Commun. math. Phys. 33 (1973) 145. 